ARTICULO ORIGINAL

\title{
Detección molecular del virus papiloma humano de alto riesgo oncogénico en muestras cervicales. Laboratorio Central de Salud Pública. Primeros Resultados
}

\section{Molecular detection of high oncogenic risk human papiloma virus in cervical samples. Central Laboratory of Public Health. First results}

\author{
*Bobadilla $\mathrm{ML}^{\mathrm{I}}$, Zorrilla ME ${ }^{\mathrm{I}}$, Villagra $\mathrm{V}^{\mathrm{I}}$, Olmedo $\mathrm{G}^{\mathrm{I}}$, Roscher $\mathrm{G}^{\mathrm{II}}$, Franco $\mathrm{F}^{\mathrm{II}}$, \\ Riveros MC $^{\text {II }}$, Gómez ME $^{\text {II }}$, Llamosas F $^{\text {III }}$ \\ ILaboratorio Central de Salud Pública (LCSP), Ministerio de Salud Púbica y Bienestar \\ Social (MSP Y BS). Asunción, Paraguay

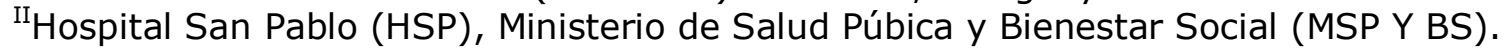 \\ Asunción, Paraguay

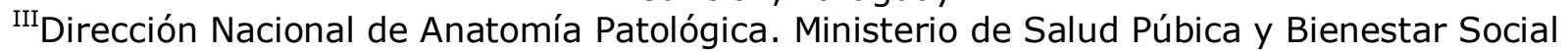
(MSP Y BS). Asunción, Paraguay

\section{RESUMEN}

El cáncer de cuello uterino es la primera causa de muerte por cáncer en mujeres en países en vías de desarrollo, con una tasa de incidencia de 34,2 por 100.000 mujeres y de mortalidad de 15,7 por 100.000 mujeres en Paraguay. La sensibilidad de la citología está entre 30-60\%, mientras que la de la detección molecular del Virus Papiloma Humano (VPH) en muestras cervicales, es mayor al 90\% para detectar neoplasia intraepitelial cervical de grado 2 (CIN II) o más. El objetivo de este trabajo fue describir la frecuencia de detección de VPH de alto riesgo (AR) y su distribución por edad en mujeres que concurrieron al Hospital San Pablo, de mayo a agosto de 2.013. Se estudiaron 170 muestras cervicales de pacientes que accedieron a participar firmando un consentimiento informado. Se utilizó el sistema Cobas 4800 HPV Test (Roche) que detecta los VPH-AR 16 y 18 , y un pool de $10 \mathrm{VPH}-A R(31,33,35,39,45,51,52,56,58,59)$ y dos de "probable" alto riesgo $(66,68)$. La frecuencia de infección por VPH-AR fue del $16 \%$, la infección decrecía con la edad y el mayor número de casos apareció en mujeres menores de 30 años. El $\mathrm{VPH}-16$ fue encontrado en todos los grupos de edades. Este es el primer reporte de la detección de ADN de VPH-AR en el LCSP, y se muestra que la prevención y control del cáncer cérvico-uterino es una prioridad de salud pública en el país por la gran carga de la enfermedad evidenciada por su alta incidencia y mortalidad.

Palabras clave: Virus Papiloma Humano, COBAS 4800 HPV test, muestras cervicales.

\section{ABSTRACT}

Cervical cancer is the leading cause of cancer death in women in developing countries, with an incidence rate of 34.2 per 100,000 women and 15.7 deaths per 100,000 women in Paraguay. The sensitivity of cytology is $30-60 \%$ while molecular detection of Human Papilloma Virus (HPV) in cervical samples is over $90 \%$ for cervical intraepithelial neoplasia grade 2 (CIN II) or more. The aim of this study was to describe the frequency of detection of high-risk HPV (HR) and their distribution by age in women who attended the Hospital San Pablo, from May to August 2013. One hundred seventy cervical samples from patients who agreed to participate by signing an informed consent. The 4800 Cobas system HPV Test (Roche), which detects the HR-HPV genotypes 16 and 18, a pool of 10

\footnotetext{
*Autor Correspondiente: Dra. María Liz Bobadilla, Laboratorio Central de Salud Pública (LCSP), Ministerio de Salud Púbica y Bienestar Social (MSP Y BS). Asunción, Paraguay Email: bobadillaml@gmail.com

Fecha de recepción: noviembre 2014; Fecha de aceptación: enero 2015
} 
HR-HPV $(31,33,35,39,45,51,52,56,58,59)$ and two "probable" high risk (66.68). The frequency of HR-HPV infection was $16 \%$, the infection decreased with age and the highest number of cases occurred in women under 30 years. HPV-16 was found in all age groups. This is the first report about detection of DNA HR-HPV in the LCSP and it showed that prevention and control of cervical cancer is a public health priority in the country by the large burden of the disease as evidenced by its high incidence and mortality.

Keywords: Human Papilloma Virus, COBAS 4800 HPV Test, cervical samples.

\section{INTRODUCCIÓN}

El cáncer de cuello uterino es el tercer tipo de cáncer más común que afecta a las mujeres en el mundo y la primera causa de muerte por cáncer en mujeres en países en vías de desarrollo. Se estiman más de 528.000 casos nuevos y 266.000 muertes al año. La tasa de incidencia estandarizada por edad en América del Sur es 24,1 por 100.000 mujeres y en Paraguay la incidencia es muy superior a las observadas en otros países vecinos, siendo la tasa de incidencia 34,2 por 100.000 mujeres y la tasa de mortalidad es de 15,7 por 100.000 mujeres (1).

Está comprobado que la infección persistente por el virus papiloma humano (VPH) es la causa necesaria para el desarrollo de lesiones intraepiteliales escamosas y del cáncer cervical. Más de 120 genotipos han sido identificados y aproximadamente 40 infectan el tracto anogenital femenino y masculino. Han sido clasificados de acuerdo con el potencial para provocar cambios malignos en el epitelio cervical en VPH de alto riesgo (VPH-AR), como VPH-16, 18, 31, 33, 35, 39, 45, 51, 52, 56, 58 y 59; VPH de probable alto riesgo (VPH-pAR) como VPH-26, 53, 66, 68, 73 y 82, y VPH de bajo riesgo (VPH-BR), como VPH-6, 11, 40, 42, 43, 44, 54, 61, 70, 72, 81 y CP6108) (2-5).

El uso de la citología cervico-vaginal convencional ha logrado reducir la mortalidad por cáncer de cuello uterino en países desarrollados $(6,7)$, tanto cuando es aplicada en programas organizados de detección como cuando se tamiza una proporción grande de mujeres de manera oportunista (8). Estudios recientes han demostrado que aún con un adecuado control de calidad, la citología convencional tiene en promedio una sensibilidad del $53 \%$ ( IC $_{95 \%} 48,6-57,4 \%$ ) en Europa y Estados Unidos (9). Sin embargo, en América Latina su uso ha tenido poco impacto en la incidencia y mortalidad de cáncer de cuello uterino, debido a la baja sensibilidad de la prueba, la baja cobertura de los programas de tamizaje y el elevado porcentaje de mujeres con anormalidades citológicas que no son evaluadas y/o tratadas adecuadamente.

En consecuencia, desde la década de los 90, se han venido estudiando pruebas de tamizaje alternativas como la inspección visual después de la aplicación del ácido acético (IVAA) y principalmente la detección del material genético del VPH. La validez y precisión de esta última han sido evaluadas extensamente en varios estudios en Latinoamérica y el mundo (10).

La prueba del ADN de los genotipos de VPH-AR en muestras cervicales tiene una sensibilidad mayor al $90 \%$ para detectar lesiones de neoplasia intraepitelial cervical de grado 2 (CIN II) o más, aunque es menos específica que la citología. Por ello, se propone esta prueba para el tamizaje en mujeres mayores de 30 años y en cohortes vacunadas $(9,11,12)$. Sin embargo, un valor importante de la prueba de ADN es su altísimo valor predictivo negativo cercano al $100 \%$ que permite espaciar los cribados con la seguridad de no desarrollar CIN III en tres años, y algunos autores aseguran hasta en cinco años $(13,14)$.

Diferentes técnicas moleculares para la detección y tipificación de VPH se han ido introduciendo en Paraguay en los últimos años. Así pues, con los métodos de PCR (Reacción en Cadena de la Polimerasa) y RFLP (Polimorfismo de Longitud de Fragmento de Restricción) se realizó un estudio en materiales de biopsias de cáncer de cuello uterino que demostró la presencia de ADN de VPH en el 73,1\% de los casos y los tipos más 
comunes fueron de 16, 18, 45, 33, 31, 52, 35 y 39. El HPV-16 era el más frecuente en carcinomas de células escamosas, mientras que el VPH 18 y 45 en tumores glandulares (15).

Otra metodología posteriormente introducida en el país fue la captura híbrida (HC II, Corporación Digene, EEUU), primera metodología aprobada por la Administración de Alimentos y Medicamentos (FDA, EEUU) para cribado primario del cáncer cervicouterino. En un reporte de Torres LM et al., se analizaron muestras cervicales utilizando el ensayo de HC II para la detección del genoma de VPH-AR. El $25 \%$ de las mujeres con citología negativa para lesión intraepitelial escamosa y el $72 \%$ de las mujeres con células escamosas atípicas de significado incierto fueron positivas para VPH-AR. Esto demuestra la alta sensibilidad de la prueba para detectar la infección incluso en tejidos de apariencia normal, corroborando que el uso de la citología junto a la HC II podría incrementar la eficiencia de los programas de tamizaje de cáncer de cuello uterino (16).

En un esfuerzo por aumentar las capacidades de detección y diagnóstico de VPH en el país, el Ministerio de Salud Pública y Bienestar Social (MSPyBS) instaló en el 2.013 la Prueba para HPV Cobas 4800 HPV Test (Roche Diagnostics, Alemania) en el Laboratorio de Salud Pública (LCSP). Este sistema ha sido validado por la FDA en el 2.011 para cribado primario del cáncer cervicouterino en mujeres mayores de 25 años y clínicamente validado por el estudio de referencia ATHENA (Addressing The Need for Advanced Diagnostics/Respondiendo a la Necesidad de un Diagnóstico Avanzado) (17).

Esta metodología presenta la principal ventaja de identificar separadamente los genotipos 16 y 18 , que han sido detectados en el $70 \%$ de las CIN III y en el $75 \%$ de los casos de cáncer cervical. Además, estudios han observado que el grupo de mujeres infectadas con los genotipos 16 y 18 tiene un riesgo mayor de presentar lesiones de alto grado, $20 \%$ y $17 \%$ respectivamente, frente al $1-2 \%$ si están infectadas con otros VPH-AR $(18,19)$. Por lo tanto, la utilización de una prueba que determine individualmente los VPH16 y 18 podría identificar un grupo de mujeres con mayor riesgo a desarrollar lesión.

El objetivo de este estudio fue describir la frecuencia de detección de VPH-AR por el Cobas 4800 HPV Test y su distribución por edad en mujeres que concurrieron al consultorio externo del Programa de Patología Cervical del Hospital San Pablo del Ministerio de Salud Pública y Bienestar Social de mayo a agosto de 2.013.

\section{MATERIALES Y MÉTODOS}

Se realizó un estudio observacional de corte transverso, en el que se incluyeron muestras cervicales pertenecientes a mujeres que acudieron al consultorio externo del Programa de Patología Cervical del Hospital San Pablo, dependiente del MSP y BS, de mayo a agosto de 2.013. A este servicio de salud, acuden normalmente las pacientes para realizarse el examen citológico cervicovaginal (PAP) que es el método de tamizaje de rutina en el país. Fueron invitadas a realizarse la prueba de VPH aquellas pacientes que cumplían con criterios de selección preestablecidos: ser sexualmente activa, no embarazada, sin histerectomía total y sin una citología previa anormal. Se excluyeron pacientes con antecedentes de anormalidades citológicas (ASCUS o más) detectados por la prueba de Papanicolau de acuerdo con los criterios de Bethesda-2.001. Las participantes firmaron un consentimiento informado y respondieron un cuestionario sobre características socio-demográficas y factores de riesgos asociados al cáncer cérvicouterino.

Los especímenes clínicos consistieron en células exfoliadas recolectadas usando un cepillo citológico para endocervix. Una vez tomada la muestra, se hizo girar el cepillo suavemente unas tres a cuatro veces para desprender las células exfoliadas dentro del vial que contiene el medio para Recolección de Células Cobas PCR (Roche Diagnostics, Mannheim, Germany). Este medio permite conservar la integridad de las muestras por un período máximo de 6 meses a temperaturas entre 2 y $30^{\circ} \mathrm{C}$. 
La detección de VPH se realizó en el LCSP con el Cobas 4800 HPV Test (Roche Diagnostics, Mannheim, Germany), aprobado por FDA en el 2.011 y que consiste en un ensayo cualitativo PCR en tiempo real totalmente automatizado que permitió la detección individual de los VPH-16 y VPH-18 y de un pool de 10 genotipos de $\operatorname{AR}(31,33,35,39$, $45,51,52,56,58$ y 59$)$ y dos de pAR (66 y 68). Este sistema está compuesto por el Cobas $x 480$, con el que se realizó la extracción y purificación simultánea de ADN del VPH y ADN celular a partir de las muestras cervicales sin tratamiento previo como indica el fabricante; y el Cobas z 480, con el cual se realizó detección del ADN de los VPH-AR mediante la amplificación de una secuencia de 200 pares de bases de la región L1 del genoma del VPH y del gen de la $\beta$-globina humana mediante la amplificación de una secuencia de 330 pares de bases usada como control interno para validación del proceso. Sondas TaqMan ${ }^{\circ}$, marcadas con cuatro diferentes fluoróforos, fueron utilizadas para la detección de los productos amplificados durante la PCR y finalmente los resultados fueron interpretados por el software como "negativo", "VPH-16", "VPH-18", "otros VPH-AR" o la combinación de los tres últimos.

Los resultados del Cobas 4800 HPV Test fueron agrupados en las siguientes categorías: a) positivos para $\mathrm{VPH}-16$, excluyendo co-infecciones con $\mathrm{VPH}-18 \mathrm{u}$ otros VPH-AR; b) positivos para $\mathrm{VPH}-18$, excluyendo co-infecciones con $\mathrm{VPH}-16 \mathrm{u}$ otros VPH-AR; c) positivos para otros VPH-AR, excluyendo co-infecciones con VPH-16 ó VPH-18; d) combinaciones de los resultados anteriores se consideraron co-infecciones y e) negativos para la detección VPH-AR. Se estudió la distribución de los distintos genotipos por tres grupos de edades: menores de 30 años, de 30-50 años y mayores de 50 años.

El protocolo de este estudio fue revisado y aprobado previamente por el Comité de Ética en Investigación del LCSP de acuerdo a lo estipulado en la Declaración de Helsinki (1.964). Los datos fueron codificados para mantener la confidencialidad de los mismos y se realizó un análisis descriptivo de las características epidemiológicas de las pacientes calculando las medias y las desviaciones estándar para las variables cuantitativas, frecuencias y porcentajes para las cualitativas utilizando herramientas estadísticas de Microsoft Excel 2.010.

\section{RESULTADOS}

Se incluyeron 170 muestras de mujeres que consultaron en el Servicio de Patología Cervical del Hospital San Pablo, entre mayo y agosto de 2.013, cumplieron con los criterios establecidos y accedieron a participar del estudio. Solo una muestra fue inválida por el sistema Cobas 4800 HPV Test por fallo de detección del control interno de $\beta$ globina, por lo que el análisis fue realizado con 169 muestras.

Las pacientes estudiadas tenían una edad promedio de $37,6 \pm 11,2$ años, encontrándose que el 56,2 \% tenía entre 30-50 años. El inicio de las relaciones sexuales fue a los $18,3 \pm 3$ años. El promedio del número de parejas sexuales fue de $2(\min 1-\max 6)$ y para la paridad fue de 3 ( $\min 1$ - máx 10$)$. El uso de anticonceptivos orales fue referido por el $17,8 \%$ de las pacientes y se declaró fumadora el $6,7 \%$ de ellas.

Teniendo en cuenta los datos globales, se pudo observar que el $16,0 \%(27 / 169)$ de las pacientes evaluadas fue positivo para VPH-AR. La Tabla 1 muestra la distribución por grupos de edad de los casos positivos para VPH-AR y puede apreciarse que la infección va decreciendo con la edad, estando el mayor número de casos en el grupo de mujeres menores de 30 años. 
Tabla 1. Distribución de la frecuencia de VPH-AR según la edad.

$\mathrm{n}=169$

\begin{tabular}{lcc}
\hline \multicolumn{1}{c}{ Edad } & No Pacientes & $\mathbf{N}^{\circ}$ VPH-AR positivo (\%) \\
\hline Menor de 30 años & 50 & $14(28,0)$ \\
$30-50$ años & 95 & $12(12,6)$ \\
Mayor de 50 años & 24 & $1(4.2)$ \\
\hline
\end{tabular}

Infecciones múltiples fueron detectadas en el $2 \%$ (4/169) de las muestras analizadas y en el $15 \%$ (4/27) de los casos positivos de VPH-AR. El VPH-16 fue encontrado en todos los grupos de edades y representó el $22 \%$ (6/27) de los casos excluyendo las coinfecciones con otros VPH-AR. El VPH-18 se encontró únicamente acompañado de otros VPH-AR, representando el 7,4\% (2/27). No fueron detectadas co-infecciones de VPH 16 y VPH 18 (Tabla 2).

Tabla 2. Distribución por edad de los genotipos de VPH-AR detectados por el Cobas 4800 HPV Test. $n=27$ mujeres positivas para infección viral

\begin{tabular}{cccccc}
\hline Edad & VPH-16 & VPH-18 & $\begin{array}{c}\text { Otros } \\
\text { VPH-AR } \\
(*)\end{array}$ & $\begin{array}{c}\text { VPH-16 } \\
+ \\
\text { Otros } \\
\text { VPH-AR }\end{array}$ & $\begin{array}{c}\text { VPH-18 } \\
+ \\
\text { Otros } \\
\text { VPH-AR }\end{array}$ \\
\hline $\begin{array}{c}\text { Menor de } 30 \\
\text { años }\end{array}$ & 3 & - & 7 & 2 & 2 \\
$30-50$ años & 2 & - & 10 & - & - \\
$\begin{array}{c}\text { Mayor de } 50 \\
\text { años }\end{array}$ & 1 & - & - & - & - \\
\hline$*$ excluyendo co-infecciones con $\mathrm{VPH}-16 \mathrm{y} \mathrm{VPH-18.}$ &
\end{tabular}

\section{DISCUSIÓN}

Este es el primer reporte de la frecuencia de detección de ADN de VPH-AR desde la implementación de la prueba en el Laboratorio Central de Salud Pública. La frecuencia de la infección por VPH-AR en las mujeres estudiadas fue del 16,0\%, similar a la descripta en otros países latinoamericanos como México, 14,5\%; Costa Rica, 16\%; Colombia, 14,8\% y Chile, $15,8 \%$ en estudios de cribado primario en pacientes sin historia previa de citología anormal $(9,20,21)$. Además, es semejante al detectado en un estudio realizado en comunidades indígenas del Paraguay, que reportó una prevalencia de $23 \%$ para cualquier tipo de VPH y de $16,1 \%$ para VPH-AR, en el que todas las pacientes estudiadas fueron negativas para lesiones escamosas intraepiteliales (22).

El mayor número de casos se encontró en los grupos de mujeres menores de 30 años y fue decreciendo con la edad, coincidiendo con lo observado en América Latina que se caracteriza por un pico antes de los 25 años, representando infecciones recientes. Sin embargo, no se presentó un segundo pico en mujeres mayores de 50 años, como se ha observado en varios estudios de la región, lo que podría ser atribuido al comportamiento sexual de las mujeres o sus compañeros o a la reactivación de infecciones latentes (9). Cabe destacar que estudios incluyendo un mayor número de muestras deben realizarse para obtener resultados más concluyentes. Se sabe que en mujeres jóvenes, menores de 
30 años, la infección por VPH, incluyendo los VPH-AR es eliminada en más del $80 \%$ de los casos. Sin embargo, se ha confirmado también que el cáncer cervical invasivo por VPH-16 y VPH-18 se presenta más en mujeres más jóvenes que los tumores malignos producidos por otros genotipos de alto riesgo (23). Debido a ello, la utilización de una prueba de detección de ADN de VPH-AR que identifique separadamente los genotipos 16 y 18 podría contribuir a identificar grupos de mujeres con mayor riesgo a desarrollar lesiones y cáncer de cuello uterino, quienes podrían recibir un seguimiento más cercano.

El sistema Cobas 4800 HPV Test utilizado en este estudio presenta la ventaja principal de identificar separadamente estos genotipos, además de otras como la facilidad del flujo de trabajo del laboratorio, la automatización de la prueba, la utilización de viales primarios para citología líquida, la inclusión de un elevado número de muestras y la ausencia de reactividad cruzada con genotipos no carcinogénicos. Es una de las metodologías de elección actualmente para los programas de tamizaje del cáncer cérvicouterino (24). No obstante, este sistema no permite genotipificar los VPH-AR no 16 y no 18, haciendo necesaria la utilización de otras metodologías de tipificación comerciales como Linear Array HPV Genotyping Test (Sistema Molecular de Roche, Alemania), Microarray (Papillocheck Greiner Bio One, Alemania); o no comerciales, como la PCR asociada a la hibridación reversa (PCR-RLB) (25).

En el año 2.013, el MSP y BS ha incorporado este método de detección como estudio piloto dentro de los programas de tamizaje y ha introducido la vacunación contra el VPH, demostrando que la prevención y control del cáncer cérvico-uterino es una prioridad de salud pública en el país debido a la gran carga de la enfermedad probada por su alta incidencia y mortalidad. El tamaño de la muestra analizada es una limitación importante en este trabajo; pero puede observarse que la frecuencia de VPH-AR coincide con lo hallado en la región. Sin embargo, se evidencia la necesidad de aumentar el número de pacientes estudiadas y realizar el cruce de los resultados con los de la citología convencional para evaluar la sensibilidad clínica de esta metodología para detectar lesiones precancerosas (11).

\section{REFERENCIAS BIBLIOGRÁFICAS}

1. International Agency for Research on Cancer. GLOBOCAN 2012: Estimated cancer incidence, mortality and prevalence worldwide in 2012. [Internet] Lyon: WHO; 2012. [citado 12 de agosto de 2014]. Disponible en http://globocan.iarc.fr/

2. Muñoz N, Castellsagué X, de Gonzalez AB, Gismann L. Chapter 1: HPV in the etiology of human cancer. Vaccine. 2006; 24 Suppl 3:S3/1-10.

3. WHO. Human papillomaviruses [Internet]. Lyon: WHO; 2007. [citado 15 de agosto de 2014]. Disponible en: http://monographs.iarc.fr/ENG/Monographs/vol90/mono90.pdf.

4. Schiffman M, Herrero R, DeSalle R, Hildesheim A, Wacholder S, Rodriguez AC, et al. The carcinogenicity of human papillomaviruses types reflects viral evolution. Virology. 2005; 337: 7684.

5. Gall SA. Female genital warts: global trends and treatments. Infect Dis Obstet Gynecol. 2001; 9: 149-54.

6. Sasieni P, Adams J. Effect of screening on cervical cancer mortality in England and Wales: analysis of trends with an age period cohort model. BJM. 1999; 318(7193): 1244-5.

7. Hakama M, Louhivuori K. A screening programme for cervical cancer that worked. Cancer Surv. 1988; 7(3): 403-16.

8. Arbyn M, Rebolj M, De Kok IM, Fender M, Becker N, O'Relly M, et al. The challenges of organizing cervical screening programmes in the 15 old member states of the European Uninon. Eur J Cancer. 2009; 45(15): 2671-8.

9. Almonte M, Murillo R, Sánchez G, Jerónimo J, Salmerón J, Ferreccio C, et al. Nuevos paradigmas y desafíos en la prevención y control del cáncer de cuello uterino en América Latina. Sal Pub Mex. 2010; 52 (6): 544-59.

10. Cortes J, Martinón-Torres F, Ramón y Cajal JM, Gil A, Velasco J, Abizanda M, et al. Prevención primaria y secundaria de los cánceres de cuello de útero y vulva: recomendaciones para la práctica clínica. Prog Obstet Ginecol. 2010; 53 (Suppl 1): 1-19. 
11. Ferreccio C, Barriga MI, Lagos M, Ibáñez C, Poggi H, González F, et al. Screening trial of human papillomavirus for early detection of cervical cancer in Santiago, Chile. Int J Cancer. 2013; 132(4):916-23.

12. Picconi MA. Detección de virus papiloma humano en la prevención del cáncer cérvico-uterino. [Internet]. Buenos Aires: Medicina; 2013. [citado 24 de agosto de 2014]; 73 (6): 585-96.

13. Wrigth TC, Schiffman M, Solomon D, Cox T, García F, Goldie S, et al. Interim guidance for the use of human papillomavirus DNA testing as an adjunct to cervical cytology for screening. Obstet Gynecol. 2004; 103(2):304-9.

14. De Sanjosé S, Quint W, Geraest D, Klaustermeir JE, Lloveras B, Tous S, et al. Human papillomavirus genotype attribution in invasive cervical cancer: a restrospective cross-sectional worldwide study. Lancet Oncol. 2010; 11(11): 1048-56.

15. Kasamatsu E, Cubilla AL, Alemany L, Chaux A, Tous S, Mendoza L, et al. Type-specific human papillomavirus distribution in invasive cervical carcinomas in Paraguay: A study of 432 cases. J Med Virol. 2012 Oct; 84(10):1628-35.

16. Torres LM, Páez M, Insaurralde A, Rodríguez MI, Castro A, Kasamatsu E. Detection of high risk human papillomavirus cervical infections by the hybrid capture in Asunción, Paraguay. Braz J Infect Dis. 2009 Jun; 13(3):203-6.

17. Wright TC, Stoler MH, Behrens CM, Apple R, Derion T, et al. The ATHENA human papillomavirus study: design, methods and baseline results. Am J Obstet Gynecol. 2012; 206(1):46e1-46e11.

18. Guardado-Estrada M, Juárez-Torres E, Román-Bassaure E, Medina-Martinez I, Alfaro A, et al. The distribution of high-risk human papillomaviruses is different in young and old patients with cervical cancer. PLoS One. 2014 Oct 8; 9(10):e109406.

19. Sanjosé S, Diaz M, Castellsagué X, Clifford G, Bruni L, Muñoz N, et al. Worldwide prevalence and genotype distribution of cervical human papillomavirus DNA in women with normal cytology: a meta-analysis. Lancet Infect Dis. 2007 Jul; 7(7):453-9.

20. Bruni L, Diaz M, Castellsagué X, Ferrer E, Bosch FX, de Sanjosé S. Cervical human papillomavirus prevalence in 5 continents: Meta-analysis of 1 million women with normal cytological findings. J Infect Dis. 2010; 202(12):1789-99.

21. Ferreccio C, Prado R, Luzoro A. Prevalencia poblacional y distribución por edad del virus papiloma humano entre mujeres en Santiago, Chile. Pontificia Universidad Católica de Chile. Boletín Escuela de Medicina. 2005; 30(1): 34-9.

22. Mendoza L, Mongelos P, Páez M, Castro A, Rodríguez-Riveros I, Giménez G, et al. Human papillomavirus and other genital infections in indigenous women from Paraguay: a cross-sectional analytical study. BMC Infect Dis. 2013 Nov 9; 13:531.

23. Mateos ML, Chacón A, Rodríguez-Domínguez M, Sanz Itziar R. Evaluación de un sistema de PCR a tiempo real (cobas 4800) para la detección separada de los genotipos 16 y 18 y otros genotipos de alto riesgo del virus del papiloma humano en la prevención del cáncer cervical. Enferm Infecc Microbiol Clin. 2011; 29(6): 411-4.

24. OPS, OMS Comunicado: La prueba de VPH como examen primario para prevenir el cáncer cervicouterino podría salvar vidas en América Latina y el Caribe. [Internet]. [citado 10 de setiembre de 2014]. Disponible en:

www.paho.org/hq/index.php?option=com_content\&view=article\&id=9570\%3Aprimary-creening-

with-hpv-test-could-save-lives-prevent-cervical-cancer\&catid=740\%3Anews-press-

releases\&Itemid $=1926 \&$ lang $=$ es

25. WHO. Human papillomavirus laboratory manual. Geneva: WHO; 2009. 